\title{
Media Naturalness and the Ability to Predict Generosity in a Give-Some - Get-Some Interaction ${ }^{1}$
}

\author{
Roderick E. White \\ Western University \\ rewhite@ivey.uwo.ca
}

\author{
Derrick J. Neufeld \\ Western University \\ dneufeld@,ivey.uwo.ca
}

\author{
Mahdi Roghanizad \\ University of Waterloo \\ mmroghan@uwaterloo.ca
}

\begin{abstract}
Evolutionary psychologists believe the human mind evolved to solve adaptive problems present in our ancestral environment. Our hominid ancestors survived in face-to-face groups by assessing the cooperative intentions of other group members. Media naturalness theory postulates face-to-face is the most 'natural' communication medium. This paper reports results from a laboratory experiment examining the ability of student subjects to predict the generosity of a counter-party under two media conditions: Face-to-Face (FtF), the more natural condition; and Video-to-Video (VtV), the less natural, technology-mediated condition. After a five-minute interaction, subjects took part in a givesome - get-some exchange and then predicted the generosity of their counterparty. Consistent with media naturalness theory, FtF subjects predicted generosity at a frequency greater than chance. Surprisingly, generosity predictions for the VtV condition were not significantly different from chance. Generosity prediction relates to important organizational behaviors such as cooperativeness, trust, and teamwork. Implications and future research opportunities are discussed.
\end{abstract}

\section{Introduction}

This paper's specific purposes are to establish and validate an experimental methodology for predicting generosity (or cooperation) in a laboratory context; and to compare the frequency of prediction accuracy under face-to-face and technology mediated, video-to-video conditions. More generally this research considers how the naturalness of communication media affects our ability to solve adaptive problems.

Effective exchange relationships are fundamental to economic prosperity [11]. The ability to identify the cooperativeness of exchange partners has the potential to greatly facilitate exchange by reducing transaction costs, thus enhancing the performance of both organizations and markets. Although the origin and role of generosity, cooperation, sharing and trust is a widely studied and much debated topic $[8,25,42,45]$, researchers generally agree that the ability to reliably detect, ex post, how partners have behaved is essential for cooperation to have evolved [13,39,40,31]. Establishing exchange relationships with novel partners can be highly advantageous, but the absence of prior experience with, or reputational information about these partners also makes these relationships especially risky. The risk is that the prospective partner will not be cooperative, and will act opportunistically to their own advantage. Accordingly, the ability to predict, ex ante, how prospective novel partners will behave in an exchange relationship would be especially valuable.

Evolutionary psychologists $[2,12,13]$ believe the human mind (like the rest of our biology) evolved by way of natural and sexual selection to generate abilities and behaviours that solved adaptive problems "posed by the physical, ecological and social environments encountered by our ancestors" [12:188]. Along these lines, "natural selection theory defines informationprocessing problems that the mind must solve, and the task ... is to uncover the nature of the algorithms that solve them" [12:190]. While there is no domain general theory for what constitutes an adaptive behaviour, modern evolutionary biology has identified domain specific adaptive problems [9]. The domain of interest for this research study is the set of adaptive problems related to group life.

Our hominid ancestors evolved in social groups. While being part of a such a group has survival advantages [41,47], it also presents a pernicious adaptive problem: free ridership. Free riders (i.e., nonco-operators) take advantage of the benefits of group life, enhancing their own genetic fitness [16], but do not share with other members or contribute to the fitness of the group. Thus the ability to identify free riders after

\footnotetext{
${ }^{1}$ The authors would like to thank Lisa Bitacola and the volunteer assistants in the Ivey Behavioural Research Laboratory, as well as the anonymous reviewers of this manuscript for their helpful assistance and feedback.
} 
the fact, or ideally to accurately predict others who will be generous (and cooperative), contributes positively to the formation and functioning of groups and to the realization of the survival advantages of group life. It is believed that human evolution has resulted in the universal cognitive ability to identify reliable (i.e., generous and cooperative) partners [2,50]. Of course this ability must be imperfect, otherwise selfish individuals would have been excluded from ancestral groups and long ago become extinct.

Impressions of a novel partner are believed to form quickly, effortlessly and subconsciously during the initial phase of an interaction [1,28,38]. Research has repeatedly demonstrated that in face-to-face interactions, people have an uncanny (though certainly imperfect) ability to intuit whether a novel partner will be generous and cooperative, or selfish and self-serving, at a probability that is significantly greater than chance $[3,4,6,7,23,24,51]$. Evolutionary theory suggests that this ability emerged in ancestral groups, where members were collocated and engaged in synchronous interactions - both verbal and nonverbal, and through nuanced speech as well as observation of facial expressions and body language. As discussed below, modern communications technologies may influence or attenuate peoples' ability to accurately assess the cooperativeness of novel partners $[5,43,52]$.

\section{Media theories and cooperativeness}

Citing theories of media richness [14] and social presence [46], Jarvenpaa and Leidner [30] observed: "computer-based communication media may eliminate the type of communication cues that individuals use to convey trust, warmth, attentiveness and other interpersonal affectations" (p. 793). This line of thinking led to the development of media naturalness theory [33], also known as the psychobiological model [32], which is based upon Darwinian evolution and evolutionary psychology. Kock [33] explained the core argument for media naturalness theory thus: "since our Stone Age hominid ancestors communicated primarily face-toface, evolutionary pressures likely have led to the development of a brain that is consequently designed for that form of communication" [33:406].

Media naturalness theory contends that communication media that more closely resemble what occurred in our ancestral environment are more "natural". Generally speaking, co-located, synchronous, face-to-face media are believed to enable the highest level of 'natural' communication [33]. Some researchers have argued that electronic media can be used to convey "rich or natural" communications. Lee [35] used a hermeneutic lens to demonstrate that the communication quality of a message (specifically, electronic mail) was not a functional property of the medium itself, but rather was something that emerged over time in a particular context as individuals actively engaged each other. Channel expansion theory argues that individual experiences and perceptions with different communication channels substantially influence an individual's perceptions of channel richness, and thus the selection and use of particular channels [10]. Media synchronicity theory identified 'conveyance' and 'convergence' processes arising from a combination of individual- and task-familiarity, with varying communication performance outcomes [18].

In contrast to media richness and media naturalness theories, these other perspectives suggest that 'mode needn't matter', and that it is feasible and common for individuals to generate and engage in 'rich' dialogue even in online mediated settings. However, they do not have much to say about how electronic media affects the ability of people with no pre-existing relationship to pick up on subtle cues and form impressions of cooperativeness. Thus arises an interesting and practically relevant question: What is the effect of technology mediated interactions on peoples' ability to correctly predict the actual generosity or cooperativeness of others with whom they will interact?

An answer to this question has relevance for both virtual markets and virtual organizations. Organizations are increasingly reliant on physically dispersed teams with members who spend little or no time meeting face to face [29], and managing others in such dispersed contexts carries unique and complex challenges and performance implications [22,28]. Engendering cooperativeness amongst individuals is central to what effective organizations do. Similarly leading Internet brands such as eBay, Amazon and Baidu are not content to provide anonymous arm's-length markets, but rather aspire to rapidly stimulate social engagement and thus foster interpersonal trust between members, who will then confidently engage in exchange relationships with one another $[38,39,44]$.

\section{Research propositions}

Because all the natural cues are present in face-toface settings, it is expected that individuals' ability to predict generous and cooperative behaviour will be higher for face-to-face than for technology-mediated interactions. However, it is not known whether, or how quickly, different forms of technology-mediation (i.e., non face-to-face communications) attenuate this ability, especially among novel partners. For example, when people first meet, are voice cues (e.g., volume, tone, expression) more salient than visual cues (e.g., facial expression, body language, attentiveness) in assessing 
trustworthiness? Kock [33] noted that speech was a costly evolutionary adaptation (e.g., involving physical positioning of the larynx low in the neck, increasing the risk of choking), and therefore must have been associated with substantial offsetting survival benefits. At the same time, DeSteno et al. [20] found that providing interpersonal visual cues, while holding voice constant, was sufficient to enhance the prediction of generosity. Therefore, it is possible that specific cues within a specific type of communication mode (e.g., voice) may be significant for some adaptive problems in some settings, and less so for others. Carefully designed experiments examining the differential effects of media naturalness upon specific adaptive problems in specific situations are required to identify the situational salience of different cues and how they are affected by technology mediation.

Our research explores whether the ability of subjects to predict the generosity of unfamiliar partners after a brief (5-minute) interaction differs between a face-toface condition $(\mathrm{FtF})$, and the equivalent high-definition audio-video condition (VtV). Consistent with evolutionary psychology's adapted mind theory $[2,12,50]$, media naturalness theory [33], and prior empirical findings $[20,24]$, we expected the frequency of FtF subjects to predict generosity to be greater than chance. It is not known whether or how quickly this ability attenuates with technology mediation. The VtV experimental context and procedures were designed to follow as closely as possible the $\mathrm{FtF}$ condition; the video quality was high definition, the image approximated life-size realism, and the audio quality was excellent. Accordingly, and consistent with media naturalness theory it was our expectation that when compared to the FtF condition the frequency of accurate predictions for the VtV condition might degrade slightly, but we still expected it to be greater than chance frequency. This reasoning led to the following hypotheses:

H1: Subjects who interact face-to-face predict generous behaviour of a non-familiar partner, at a frequency greater than chance.

$\mathrm{H} 2$ : Subjects who interact via high definition audiovideo predict generous behaviour of a non-familiar partner, at a frequency greater than chance.

H3: Subjects who interact via high definition audiovideo predict generous behaviour of a non-familiar partner at a lower frequency than those who interact face-to-face.

\footnotetext{
2 Experimental video available online:

Video 1: http://bit.ly/1TvpXAL

Video 2: http://bit.ly/1TzHPgb

Video 3: http://bit.ly/1WOg7QN
}

\section{Design and method}

Data were collected through the use of a laboratory experiment, using student subjects recruited from the University's standard research study pool. For the FtF treatment, 114 subjects were scheduled to arrive at the behavioural laboratory in pairs, and each pair was taken to a room and asked: "Do you know each other?" Four pairs indicated familiarity; in each of these cases, the subjects were thanked, paid $\$ 6$, and dismissed from the study. Familiarity was further validated in a final survey with the question: "What is your relationship with the person you interacted with?". Subjects who indicated, "We know each other casually" or "We know each other quite well" were removed from further analysis. Several additional subjects failed to properly complete the exit survey. As a result of these exclusions, the final dataset contained 98 valid $\mathrm{FtF}$ subjects.

The research assistant (RA) handed each subject a five-dollar bill and a one-dollar coin, for a total of six Canadian dollars, and thanked them for participating. The RA then directed the subjects to view a brief introductory video describing the first of two games they would be playing ${ }^{2}$. In the first video, subjects were instructed that they would play a coin-toss game followed by a five-minute conversation, and that another monetary exchange would then follow the initial coin-toss game. They were given no specifics about the follow-on exchange, and were asked not to speculate about it during their five-minute conversation, in order to avoid the possibility of collusion. All subjects followed these instructions.

Following the first set of video instructions, the subjects played a simple coin-toss game. Each person was handed an additional two-dollar coin and instructed to verbally predict whether their own coin toss would result in a head or tail. To win this game, a subject had to be correct about their self-prediction, while the other subject had to be incorrect about their self-prediction (e.g., if Subject A predicted heads and flipped heads, while Subject B predicted tails and flipped heads, then Player A would win; if both self-predictions were correct, or both were incorrect, the subjects flipped repeatedly until there was a single winner). Once a winner was established, the RA immediately picked up both coins, handed them to the winner, and said, "Congratulations". The coin-toss game ostensibly provided a social icebreaker; however, the underlying objective of this priming manipulation was to demonstrate that there was going to be real money at 
risk, and to introduce a spirit of competitiveness into the experimental context.

Subjects were then given five minutes to interact, "to get to know one another". They were instructed to talk about any topic of their choosing, such as "your favourite courses, what sports you like, what you did last summer, et cetera". The RA left the room during this interaction, but a video camera recorded the interaction. At the end of the five-minute period, the RA returned, and one subject was taken to a separate room. Once separated, both subjects viewed a second video that described a "give-some - get-some" game, wherein they were asked to decide whether and how much money to give to the other subject. Their options were to give $\$ 0$, $\$ 2, \$ 4$ or $\$ 6$, and any amount they chose to give to the other player would be "magically doubled" - so for example, if they gave up $\$ 6$, the other subject would receive $\$ 12$. Alternatively, if they gave $\$ 0$, the other subject would receive nothing. Meanwhile, they were told, the other subject was making the same decision about giving money to them.

This payment structure was established after conducting preliminary, pre-experimental tests to establish a relevant range of payouts for this experimental subject pool. Likewise, the choice to give subjects their initial \$6 payment in the form of a fivedollar bill and a one-dollar coin was done to separate, in the subjects' minds, the $\$ 6$ as general payment for participation, from their decision to give up to $\$ 6$ in the give-some - get-some game. We also reasoned that giving them "paper money" (the $\$ 5$ bill) upfront might cause them to think twice about giving away their money, versus giving them "change" in the form of three two-dollar coins, and that the use of coins might imply that the experimenters wanted them to give some of those coins, and thus trigger an undesirable expectancy bias for giving. Subjects were told that the RA could provide change if requested. The payment amount decided by the subject was placed in an envelope, and doubled by the RA in front of the subject who gave it.

The altruistic approach of giving the maximum amount, if followed by both subjects, would maximize the net group payoff - i.e., if both gave $\$ 6$, each would receive $\$ 12$ for a joint payoff of $\$ 24$. The optimal egocentric strategy was to keep the $\$ 6$, give $\$ 0$, and accurately predict receipt of $\$ 12$ from the other subject, in which case the net group payoff would be $\$ 18-$ i.e., $\$ 0$ to the generous player and $\$ 18$ to the non-generous player. However, if both subjects followed the egocentric strategy and kept their \$6, the net group payoff would be just $\$ 12$.

Finally, and most importantly, before receiving any payment amount each subject viewed a third and final video that asked them to predict how much the other player had given them $-\$ 0, \$ 2, \$ 4$ or $\$ 6$ (which would be doubled). Subjects were informed that a correct prediction of the other subject's decision would be rewarded with an additional $\$ 8$ payment. Providing this substantial payment for a simple prediction task was intended to stimulate serious consideration about the other subject's generosity. Several additional survey questions were included to capture demographic and personality information, and to re-confirm that the subjects were not acquainted.

The RA adjusted the final (envelope) amounts to provide a minimum payment of $\$ 8$ to every subject. This was done to mask the amount of the actual payment from recipients, so that a given subject would not be able to attribute their final payment to money given by the other subject versus their having correctly predicted the other subject's decision, and thus to protect participant identities and prevent possible hard feelings. To avoid the possibility of social awkwardness care was taken to insure that participants did not encounter one another as they exited the behavioural laboratory.

The VtV design was identical to that of the FtF condition, with the exception of video-conferencing mediation. Subjects arriving to take part in the experiment were brought to separate waiting areas, and then led into separate rooms that were connected by video-conferencing equipment (i.e., a 60-inch highdefinition television monitor, and a high definition audio-video camera). Each subject was directed to sit in a chair that was placed in front of the television monitor, with a camera positioned to provide an upper-body shot (head to waist). Each subject independently watched an explanatory video, the video cameras were turned on, and the RA asked if subjects knew one other or had ever spoken. Three pairs of subjects were familiar with one another, and these subjects were paid $\$ 6$ and dismissed. Five additional subjects were removed due to either indicating familiarity on the exit survey, or because they failed to complete the exit survey. As a result of these exclusions, the final dataset contained 107 valid VtV subjects.

$\mathrm{VtV}$ subjects then completed the same procedures as the FtF subjects:

1. They played the coin-toss game, virtually from each other but in the presence of the RAs, and one winner was established and paid.

2. They took part in a five-minute videoconferencing interaction (in the absence of the RAs, but the recording was captured).

3. The cameras were turned off, they viewed the second video, and then they made their givesome - get-some decision.

4. They viewed the third video and made generosity predictions of the other subject.

5. Finally, payments were calculated and 
distributed, and the subjects were dismissed. Setup of the experimental conditions are shown in Figure 1a for $\mathrm{FtF}$ and Figure $1 \mathrm{~b}$ for $\mathrm{VtV}$ (faces are blurred for privacy reasons).

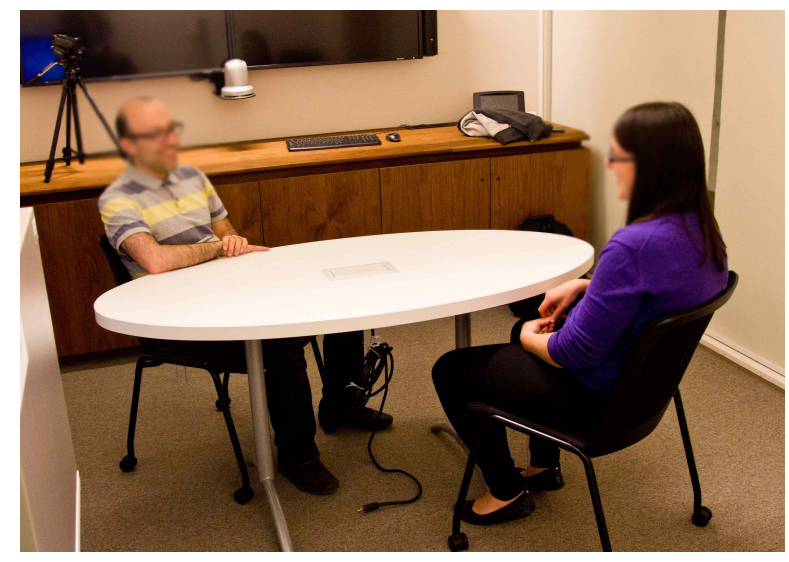

Figure 1a. Face-to-face room setup

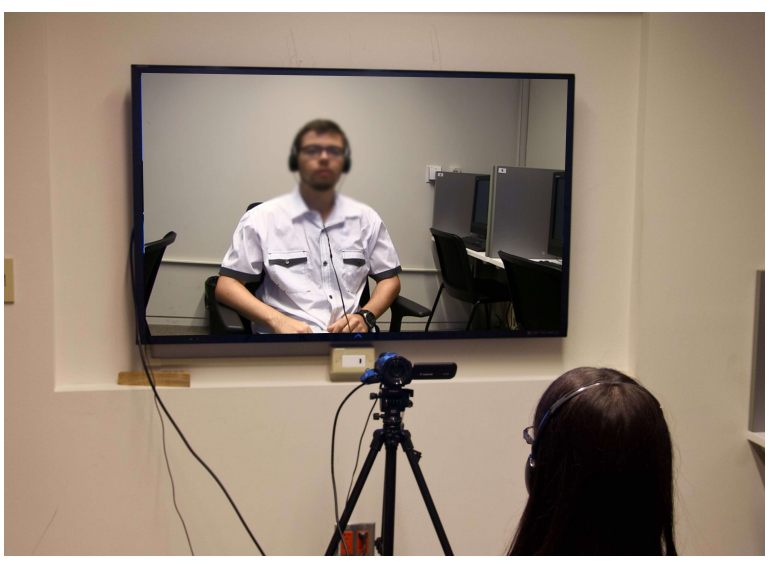

Figure 1b. Video-to-video room setup

Prior research has shown that the ability of individuals to detect generosity can be sensitive to the experimental design. Manson et al. [36:2] noted:

"In most experimental protocols, participants are informed of their impending social dilemma decision before conversing, and are (1) instructed not to discuss the game [e.g., 15] or else (2) allowed to discuss and 'disclose' their game-play decisions [e.g., 24]. The former must make for awkward social interactions, while the latter transforms the 'defector detection' challenge into the rather different task of 'liardetection' [19]."

\footnotetext{
${ }^{3}$ Using a different experimental design, Frank (2004) reports females predict more accurately than males.
}

Experimental studies with a 'get-to-know-you' conversation followed by an unannounced cooperatedefect type exchange, such as the present study, are designed to test for 'cooperation detection' and to avoid the 'liar detection' trap. These types of studies are rare.

\section{Results}

Among the 205 valid subjects participating in this study, $96 \%$ were $21-25$ years of age, $52 \%$ were male, and $48 \%$ were female (one gender response in the FtF sample was unreported). Gender ratios for the FtF (VtV) conditions were $60.2 \%(43.9 \%)$ male and $38.7 \%$ $(56.1 \%)$ female. While the subjects were assigned to treatment conditions as they arrived at the behavioural laboratory females were slightly underrepresented in the $\mathrm{FtF}$ condition and over represented in the $\mathrm{VtV}$ condition. This raised the possibility of gender differences explaining (some of) the difference between the two conditions [23]. ${ }^{3}$ However, analysis of the data uncovered no significant interaction between communication medium and gender upon prediction accuracy (ordinal logistic regression $\beta=0.39, S E=.57$, $p=0.49$ ). There was no significant difference in prediction accuracy between males and females for $\mathrm{FtF}$ to $\mathrm{VtV}$ conditions.

Actual and predicted distributions of amounts given are shown for FtF (Table 1a) and VtV (Table 1b). The average actual amount given by the $\mathrm{FtF}$ group $(M=4.49$, $S D=1.77)$ did not differ from that of the $\operatorname{VtV}$ group $(M=$ 4.47, $\left.S D=1.54 ; F_{(1,204)}=0.01, p=.92\right)$. Likewise the average predicted amount given by the $\mathrm{FtF}$ group $(M=$ 4.04, $S D=1.93)$ did not differ from that of the $\mathrm{VtV}$ group $\left(M=4.07, S D=1.65 ; F_{(1,204)}=0.02, p=.89\right)$.

\section{Table 1a. Face-to-face}

\begin{tabular}{|c|c|c|c|c|c|}
\hline & \multicolumn{4}{|c|}{ Predicted Give } & \multirow[b]{2}{*}{ total } \\
\hline & $\$ 0$ & $\$ 2$ & $\$ 4$ & $\$ 6$ & \\
\hline$\$ 0$ & 1 & 2 & 0 & 0 & 3 \\
\hline$\$ 2$ & 3 & 11 & 6 & 0 & 20 \\
\hline$\$ 4$ & 0 & 8 & 15 & 2 & 25 \\
\hline$\$ 6$ & 2 & 5 & 5 & 38 & 50 \\
\hline total & 6 & 26 & 26 & 40 & 98 \\
\hline
\end{tabular}

The diagonal cells from $\$ 0-\$ 0$ to $\$ 6-\$ 6$ reflect cases in which predicted amounts exactly matched actual amounts (i.e., there was no prediction error). For the FtF condition (Table 1a), 65 out of 98 or $66.3 \%$ of the predictions were exact matches. For the $\mathrm{VtV}$ condition (Table 1b), 35 out of 107 or $32.7 \%$ were exactly 
accurate.

Table 1b. Video-to-video

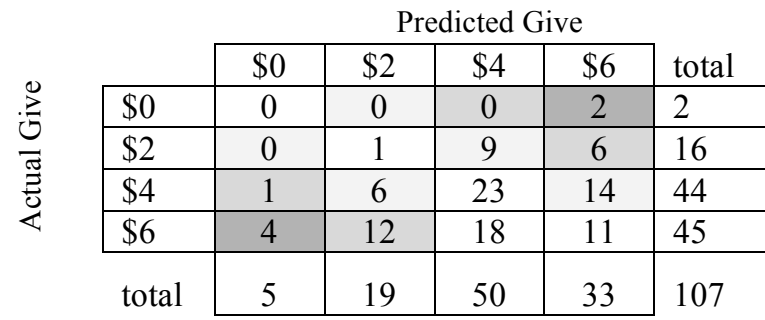

Our first hypothesis states that subjects who interact face-to-face predict generous behaviour of an unfamiliar partner, at a frequency greater than chance. To test this hypothesis, we calculated actual error values for each subject in the FtF condition (i.e., the actual amount their partner gave minus the amount they predicted would be given). The resulting values ranged from +6 (i.e., actual $\$ 6$ - predicted $\$ 0$ ) to -6 (i.e., actual $\$ 0$ - predicted $\$ 6$ ). Then, based on observed frequencies of actual amounts given (i.e., \$0: $3.1 \%$, $\$ 2: 20.4 \%$, $\$ 4: 25.5 \%$, $\$ 6: 51 \%$ ), the random error distribution was produced. Next, a Kolmogorov-Smirnov (K-S) test was used to compare the actual and random error distributions. Results showed that FtF participants' predictions were significantly more accurate than chance frequency $(p<0.01$, one-tailed), supporting our first hypothesis.

Our second hypothesis states that subjects who interact via high definition audio-video predict generous behaviour of an unfamiliar partner, at a frequency greater than chance. Following the same procedure as with the FtF sample we tested this hypothesis by calculating actual error values for each subject in the $\mathrm{VtV}$ condition (i.e., actual amount given minus predicted amount given), resulting in an error array from +6 to -6 . Then based on observed frequencies of actual amounts given (i.e. $\$ 0: 1.9 \%, \$ 2: 14.9 \%, \$ 4: 41.1 \%$, $\$ 6$ : $42.1 \%$ ), the random error distribution was generated. The K-S test was employed to compare the actual and random error distribution, and results showed no difference between the two distributions $(p>0.2$, onetailed). Thus $\mathrm{H} 2$ was not supported. Unlike those in the FtF sample, subjects interacting in the $\mathrm{VtV}$ condition were unable to predict generous behaviours of their unfamiliar partners at a frequency significantly greater than chance.

Our third hypothesis states that subjects who interact via high definition audio-video predict generous behaviour of an unfamiliar partner lesser frequency than those who interact face-to-face. Similar to the procedures used to test $\mathrm{H} 1$ and $\mathrm{H} 2$ the K-S test was employed to contrast the $\mathrm{FtF}$ prediction error distribution and $\mathrm{VtV}$ prediction error distribution. Supporting our third hypothesis, the frequency of accurate predictions was lower for $\mathrm{VtV}$ than for $\mathrm{FtF}$ and the two distributions were significantly different from one another $(p<0.01$, one-tailed).

As we considered these results and contemplated how people go about predicting the generosity of others, we reasoned that our subjects might be relying on a simple decision rule: "The counterparty will give me what I gave them". Prior research has shown that people frequently use themselves as an anchor or reference point, and that their assessment of others is essentially egocentric [21,26,27,40]. Moreover, prior research has shown that egocentrism is stronger in technology mediated communications versus face-to-face, and associated with increased private self-awareness [37], more uninhibited, unregulated and self-focused behaviour [31], and higher levels of egocentrism [34].

To better understand the differences in generosity prediction efficacy between the $\mathrm{FtF}$ and $\mathrm{VtV}$ groups, and the possible role played by egocentrism, a further post hoc analysis was performed. An ordinal logistic regression was conducted with predicted amount (i.e., the amount Subject A predicted that Subject B would give them) as the dependent variable, and two independent variables: (1) shared amount (i.e., the amount that Subject A gave to Subject B), and (2) media condition (i.e., FtF or VtV). Results revealed significant interactions between shared amount and media (the most conservative Wald statistic was $10.62, p<.001)$. To unpack the interaction effect, the data were split on the media condition, and independent Spearman's correlation analyses were conducted for each subset. We found that the predicted amount was not associated with shared amount in the FtF condition $(r=-.05, p=.62)$, while in the VtV condition there was a significant positive correlation $(r=.77, p<.001)$. These results support the proposition that in the technology-mediated $\mathrm{VtV}$ condition, subjects predicted others' behaviour largely based on their own behavior, without regard to clues they may have received via the relatively rich media interaction with their counterparty.

The foregoing analysis describes how VtV subjects made their predictions, and why the frequency of these predictions were no better than chance. It also suggests a theoretical rationale that requires further consideration. However, it does not explain how FtF subjects were able to beat the odds and make better than chance frequency predictions. Answers to this important question require further empirical study and theory application and development.

\section{Discussion}

The findings from this study show that FtF subjects had a significantly better than chance frequency of predicting the generosity of their partner, while the VtV 
subjects did not, and that the difference between the predictive capacities of the two conditions was highly significant.

The strength of these results was surprising to the researchers, considering the high quality of the audiovideo media used (i.e., the video-conferencing technology was superior to the typical "Skype" audiovideo conferencing in terms of factors such as screen size, picture resolution, sound clarity, lack of audiovideo latency lags, etc.), and the fact that the VtV experimental conditions closely replicated those of the FtF condition. The mediating technology allowed research subjects to see and hear one another with excellent imagery and sound, and yet their predictive abilities were significantly different.

When designing this study, the researchers were prepared to explore multiple conditions in addition to $\mathrm{VtV}$, such as Skype-quality video/audio, audio-only and text-only, in order to be able to observe the attenuation effects predicted by media naturalness theory. However, results from this first study suggested that any form of mediation, even those as close to natural as current technology allows, may have an immediate and consequential effect on the efficacy of generosity prediction of an unfamiliar partner.

These results open up a variety of new research questions and opportunities as described in the next section. However, this research also has immediate applicability. Understanding that the ability of unfamiliar individuals to predict generosity and cooperativeness is negatively attenuated by even high quality technology mediation has important implications for organizations making use of these technologies and relying on virtual teams and distributed leadership structures. It suggests that dispersed virtual team members, who do not have an opportunity to meet face-to-face, may be less able to assess and establish the cooperativeness of their team members with potential negative implications for team performance. Likewise, leader-follower relationships in geographically dispersed organizations that preclude face-to-face interaction may result in a lessened capacity to accurately assess the co-cooperativeness of others. We do not know if an initial FtF interaction is sufficient to overcome this assessment deficit, and if subsequent interaction can be effectively mediated by technology, or if the ability to accurately predict generosity requires $\mathrm{FtF}$ interaction prior to every cooperative act. Additional carefully designed experiments will be required to address these important questions.

For operators of e-commerce marketplaces, these results suggest that establishing a video connection between unfamiliar buyers and sellers is unlikely to enhance their ability to assess the cooperativeness of their prospective counterparty. There are also important potential implications for banks as they embrace the fintech revolution and transition their retail and commercial customers from face-to-face relationships to technology-mediated, or technology only relationships.

\section{Limitations and future research}

This study has several methodological strengths. The dependent variable, prediction error, is objective. Subjects were asked to predict the giving behaviour of the person with whom they had interacted. They were not asked their subjective opinion about how cooperative or generous they felt that person might be; instead their prediction accuracy was calculated based on an actual behavior. A related methodological strength was the inclusion of the "coin toss game", adopted during experimental pretesting as a means of triggering a competitive context. When examining this type of phenomenon, it is critical to find the right balance of priming triggers that will reveal a range of both generous and selfish behaviours [48,49]. Another important procedural step was to remove subjects who indicated they knew one another, since relational and reputational foreknowledge can significantly moderate the effects of media use and information richness [35]. By restricting the sample to include only strangers, subject predictions precluded assessments based on prior information.

The subjects in this study were undergraduate students and the normal caveats about generalizing to other sub-populations apply. However, other research suggests the ability to predict generosity may be a human universal that is innate and/or learned prior to adulthood. Work by Cosmides and her collaborators on cheater detection (the flipside of generosity prediction) has found this ability across diverse cultural groups $[2,12,13]$. If this type of ability is truly universal the relationship between media naturalness and generosity prediction would not differ amongst different human sub-populations. That said, the possibility of cultural differences cannot be dismissed without replicating this type of study across different cultures.

It is possible that with more personal experience using technology-mediated communication an individual's ability to predict generosity may improve, consistent with media use theories $[10,18]$. Future research replicating this study but using subjects with high computer literacy and substantial video interviewing experience is needed to address this 
question. $^{4}$

An important question remains: What caused the difference in $\mathrm{FtF}$ versus $\mathrm{VtV}$ generosity prediction efficacy in a tightly-controlled experimental context that was otherwise almost identical? One possible explanation involves the emission and interpretation of cues. Perhaps the frequency of salient cues emitted by the subjects differed across the two conditions. Or, the interpretation of cues in the '3-dimensional' FtF setting may have differed from that in the '2-dimensional' VtV setting. Our next step will be to explore this possibility for these subjects. Using a procedure similar to that described by DeSteno et al. [20], we will examine video recordings of these subjects' interactions, in both FtF and $\mathrm{VtV}$ conditions, to determine whether frequency of non-verbal cues associated with prediction efficacy differed.

We will also explore other possible differences between the $\mathrm{FtF}$ and the $\mathrm{VtV}$ condition (e.g., content analysis of language used). If further research on $\mathrm{FtF}$ and $\mathrm{VtV}$ media finds an absence of differences in the information transmitted, it leads towards the conclusion that the context activates, or fails to activate, an evolved generosity prediction cognitive module (i.e., in a binary, or perhaps stepwise fashion).

Media naturalness theory and evolutionary social psychology theories both suggest our brains evolved to solve social problems in a rich $\mathrm{FtF}$ communications context. Anything less than FtF conditions may fail to trigger this evolved human social cognitive module [17]. Additional experimentation and analyses is required to examine under what conditions this cognitive ability is activated, and how these conditions influence the efficacy of generosity prediction.

\section{Conclusions}

This study has a strong theoretical foundation in media naturalness theory and evolutionary psychology. The ability to predict generosity or cooperativeness addresses a well-recognized adaptive problem for ancestral and contemporary social groups: how to detect uncooperative individuals, or opportunistic free riders who are unlikely to help others or contribute to the good of the group. The theory suggests this ability is largely innate and unconscious. While our research does not prove the innateness of this ability, the FtF subjects were able to make more accurate than chance predictions after a relatively brief five-minute, unstructured interaction, while $\mathrm{VtV}$ subjects were not. The findings suggest that an innate psychobiological process may be operating - a process that functions effectively only in a

\footnotetext{
${ }^{4}$ We thank an anonymous reviewer for this suggestion.
}

natural face-to-face-setting, but is absent in technologymediated communications.

The results of this study are significant and consequential. They suggest that any form of technology mediation may substantially reduce, or even eliminate the ability of novel partners to predict cooperativeness, at a frequency greater than chance. As such, there may be no effective substitute for face-to-face interactions.

\section{References}

[1] Andersen, S.M., Reznik, I., and Manzella, L.M. Eliciting facial affect, motivation, and expectancies in transference: Significant-other representations in social relations. Journal of Personality and Social Psychology 71, 6 (1996), 1108 1129.

[2] Barkow, J.H., Cosmides, L., and Tooby, J. The Adapted Mind: Evolutionary Psychology and the Generation of Culture. Oxford University Press, 1995.

[3] Berry, D.S. and Brownlow, S. Were the physiognomists right? Personality and Social Psychology Bulletin 15, 2 (1989), 266-279.

[4] Berry, D.S. and McArthur, L.Z. Perceiving character in faces: The impact of age-related craniofacial changes on social perception. Psychological Bulletin 100, 1 (1986), 318.

[5] Bos, N., Olson, J., Gergle, D., Olson, G., and Wright, Z. Effects of four computer-mediated communications channels on trust development. Proceedings of the SIGCHI Conference on Human Factors in Computing Systems, ACM (2002), 135-140.

[6] Brosig, J. Identifying cooperative behavior: Some experimental results in a prisoner's dilemma game. Journal of Economic Behavior \& Organization 47, 3 (2002), 275290.

[7] Brownlow, S. Seeing is believing: Facial appearance, credibility, and attitude change. Journal of Nonverbal Behavior 16, 2 (1992), 101-115.

[8] Burt, R.S. and Knez, M. Trust and Third-Party Gossip. In Trust in Organizations: Frontiers of Theory and Research. SAGE, 1996.

[9] Buss, D.M. Personality and the adaptive landscape: The role of individual differences in creating and solving social adaptive problems. In The Evolution of Personality and Individual Differences. Oxford University Press, New York, 2010, 29-58. 
[10] Carlson, J.R. and Zmud, R.W. Channel expansion theory and the experiential nature of media richness perceptions. Academy of Management Journal 42, 2 (1999), 153-170.

[11] Coase, R.H. The Nature of the Firm. Economica 4, 16 (1937), 386-405.

[12] Cosmides, L. The logic of social exchange: Has natural selection shaped how humans reason? Studies with the Wason selection task. Cognition 31, 3 (1989), 187-276.

[13] Cosmides, L. and Tooby, J. Cognitive adaptations for social exchange. The Adapted Mind, (1992), 163-228.

[14] Daft, R.L., Lengel, R.H., and Trevino, L.K. Message equivocality, media selection, and manager performance: Implications for information systems. MIS Quarterly, (1987), 355-366.

[15] Dawes, R.M., McTavish, J., and Shaklee, H. Behavior, communication, and assumptions about other people's behavior in a commons dilemma situation. Journal of Personality and Social Psychology 35, 1 (1977).

[16] Dawkins, R. The Selfish Gene. Oxford University Press, 2006.

[17] Decety, J. and Sommerville, J.A. Shared representations between self and other: A social cognitive neuroscience view. Trends in Cognitive Sciences 7, 12 (2003), 527-533.

[18] Dennis, A.R., Fuller, R.M., and Valacich, J.S. Media, tasks, and communication processes: A theory of media synchronicity. MIS Quarterly 32, 3 (2008), 575-600.

[19] DePaulo, B.M., Lindsay, J.J., Malone, B.E., Muhlenbruck, L., Charlton, K., and Cooper, H. Cues to deception. Psychological Bulletin 129, 1 (2003), 74-118.

[20] DeSteno, D., Breazeal, C., Frank, R.H., et al. Detecting the trustworthiness of novel partners in economic exchange. Psychological Science, (2012), 1-8.

[21] Epley, N., Keysar, B., Van Boven, L., and Gilovich, T. Perspective taking as egocentric anchoring and adjustment. Journal of Personality and Social Psychology 87, 3 (2004), 327-339.

[22] Fang, Y. and Neufeld, D. Understanding sustained participation in open source software projects. Journal of Management Information Systems 25, 4 (2009), 9-50.

[23] Frank, R. Can Cooperators Find One Another? (Ch.2). In What Price the Moral Ground? Ethical Dilemmas in Competitive Environments. Princeton University Press, Princeton, NJ, 2004, 28-44.
[24] Frank, R.H., Gilovich, T., and Regan, D.T. The evolution of one-shot cooperation: An experiment. Ethology and Sociobiology 14, 4 (1993), 247-256.

[25] Fukuyama, F. Trust: The Social Virtues and the Creation of Prosperity. Simon and Schuster, 1996.

[26] Gilovich, T., Medvec, V.H., and Savitsky, K. The spotlight effect in social judgment: an egocentric bias in estimates of the salience of one's own actions and appearance. Journal of Personality and Social Psychology 78, 2 (2000), 211-222.

[27] Gilovich, T., Savitsky, K., and Medvec, V.H. The illusion of transparency: Biased assessments of others' ability to read one's emotional states. Journal of Personality and Social Psychology 75, 2 (1998), 332-346.

[28] Howell, J.M., Neufeld, D.J., and Avolio, B.J. Examining the relationship of leadership and physical distance with business unit performance. The Leadership Quarterly 16, 2 (2005), 273-285.

[29] Huang, L. and Murnighan, J.K. What's in a name? Subliminally activating trusting behavior. Organizational Behavior and Human Decision Processes 111, 1 (2010), 6270.

[30] Jarvenpaa, S.L. and Leidner, D.E. Communication and trust in global virtual teams. Journal of Computer-Mediated Communication 3, 4 (1998), 791-815.

[31] Kiesler, S., Siegel, J., and McGuire, T.W. Social psychological aspects of computer-mediated communication. American Psychologist 39, 10 (1984), 1123-1134.

[32] Kock, N. The psychobiological model: Towards a new theory of computer-mediated communication based on Darwinian evolution. Organization Science 15, 3 (2004), $327-348$.

[33] Kock, N. Information systems theorizing based on evolutionary psychology: An interdisciplinary review and theory integration framework. MIS Quarterly, (2009), 395 418.

[34] Kruger, J., Epley, N., Parker, J., and Ng, Z.-W. Egocentrism over e-mail: Can we communicate as well as we think? Journal of Personality and Social Psychology 89, 6 (2005), 925-936.

[35] Lee, A.S. Electronic mail as a medium for rich communication: An empirical investigation using hermeneutic interpretation. MIS Quarterly, (1994), 143-157.

[36] Manson, J.H., Gervais, M.M., and Kline, M.A. Defectors cannot be detected during "small talk" with strangers. PloS One 8, 12 (2013), 1-12. 
[37] Matheson, K. and Zanna, M.P. The impact of computermediated communication on self-awareness. Computers in Human Behavior 4, 3 (1988), 221-233.

[38] McKnight, D.H., Choudhury, V., and Kacmar, C. Developing and validating trust measures for e-commerce: An integrative typology. Information Systems Research 13, 3 (2002), 334.

[39] McKnight, D.H., Cummings, L.L., and Chervany, N.L. Initial trust formation in new organizational relationships. Academy of Management Review 23, 3 (1998), 473-490.

[40] Nickerson, R.S. How we know-and sometimes misjudge - what others know: Imputing one's own knowledge to others. Psychological Bulletin 125, 6 (1999), 737-759.

[41] Pierce, B.D. and White, R. The evolution of social structure: Why biology matters. Academy of Management Review 24, 4 (1999), 843-853.

[42] Ridley, M. The Origins of Virtue: Human Instincts and the Evolution of Cooperation. Penguin, 1998.

[43] Rocco, E. Trust breaks down in electronic contexts but can be repaired by some initial face-to-face contact.

Proceedings of the SIGCHI conference on Human factors in computing systems, ACM Press/Addison-Wesley Publishing Co. (1998), 496-502.

[44] Roghanizad, M.M. and Neufeld, D.J. Intuition, risk, and the formation of online trust. Computers in Human Behavior 50, (2015), 489-498.

[45] Rousseau, D.M., Sitkin, S.B., Burt, R.S., and Camerer, C. Not so different after all: A cross-discipline view of trust. Academy of Management Review 23, 3 (1998), 393-404.
[46] Short, J., Williams, E., and Christie, B. The social psychology of telecommunications. (1976).

[47] Sober, E. and Wilson, D.S. Unto Others: The Evolution and Psychology of Unselfish Behavior. Harvard University Press, 1999.

[48] Stewart, A.J. and Plotkin, J.B. From extortion to generosity, evolution in the iterated prisoner's dilemma. Proceedings of the National Academy of Sciences 110, 38 (2013), 15348-15353.

[49] Stewart, A.J. and Plotkin, J.B. Collapse of cooperation in evolving games. Proceedings of the National Academy of Sciences 111, 49 (2014), 17558-17563.

[50] Tooby, J. and Cosmides, L. On the universality of human nature and the uniqueness of the individual: The role of genetics and adaptation. Journal of Personality 58, 1 (1990), 17-67.

[51] Whitener, E.M., Brodt, S.E., Korsgaard, M.A., and Werner, J.M. Managers as initiators of trust: An exchange relationship framework for understanding managerial trustworthy behavior. Academy of Management Review 23, 3 (1998), 513-530.

[52] Wilson, J.M., Straus, S.G., and McEvily, B. All in due time: The development of trust in computer-mediated and face-to-face teams. Organizational Behavior and Human Decision Processes 99, 1 (2006), 16-33. 